\title{
Relationship between electronic structures and antiplasmodial activities of xanthone derivatives: a 2D-QSAR approach
}

\author{
Gaston A. Kpotin ${ }^{1}$. Affoué Lucie Bédé ${ }^{2}$ - Alice Houngue-Kpota ${ }^{1}$. Wilfried Anatovi ${ }^{1}$. Urbain A. Kuevi ${ }^{1}$. \\ Guy S. Atohoun ${ }^{1}$ - Jean-Baptiste Mensah ${ }^{1}$. Juan S. Gómez-Jeria ${ }^{3} \cdot$ Michael Badawi $^{4}$ (D)
}

Received: 19 February 2019 / Accepted: 1 April 2019 / Published online: 24 May 2019

(C) Springer Science+Business Media, LLC, part of Springer Nature 2019

\begin{abstract}
Malaria is an important disease causing many death in several countries of Africa and Asia. In these continents, some plants such as Garcinia cola are used to fight against this disease because they contain xanthone derivatives which present antiplasmodial activity. The present theoretical study aims to establish a relationship between the electronic structure and the antiplasmodial activity of some xanthone derivatives, and more specifically to build a 2D-pharmacophore model in order to predict the biological activity of xanthone derivatives. The calculations are performed within the density functional theory (DFT) using the B3LYP/6$31 \mathrm{G}(\mathrm{d}, \mathrm{p})$ level of theory. The developed approach quantitative structure-activity relationship (QSAR) follows the KlopmanPeradejordi-Gómez (KPG) methodology. We obtain a statistically significant equation relating the variation of the logarithm of half maximal inhibitory concentration $\left(\log \left(\mathrm{IC}_{50}\right)\right)$ with the variation of the numerical values of a set of eight local atomic reactivity descriptors $\left(R=0.98, R^{2}=0.97\right.$, adj- $R^{2}=0.95, F(8.13)=48.63, p<0.00000$, SD 0.08$)$. The antiplasmodial activity seems to be driven by atomic orbitals and charges. Our 2D-pharmacophore model should be useful to propose new xanthone derivatives with higher antiplasmodial activity.
\end{abstract}

Keywords Xanthone $\cdot$ Antiplasmodial $\cdot$ QSAR $\cdot$ DFT $\cdot$ Klopman-Peradejordi-Gómez approach $\cdot$ Malaria

\section{Introduction}

Malaria is one of the most fatal diseases in the developing countries of Africa. In 2016, 91 countries reported a total of 216 million cases of malaria, an increase of 5 million cases over the previous year. The global tally of malaria deaths reached

Gaston A. Kpotin

gaston.kpotin@fast.uac.bj

$\triangle$ Michael Badawi

michael.badawi@univ-lorraine.fr

1 Laboratory of Theoretical Chemistry and Molecular Spectroscopy, Faculty of Sciences and Techniques, University of Abomey - Calavi, 03 BP 3409 Cotonou, Benin

2 Laboratoire de Chimie Organique Structurale, Université Félix Houphouët-Boigny, 22 BP 582, Abidjan, Côte d'Ivoire

3 Quantum Pharmacology Unit, Department of Chemistry, Faculty of Sciences, University of Chile, Las Palmeras 3425, Nuñoa, 7800003 Santiago, Chile

4 Laboratoire de Physique et Chimie Théoriques, Université de Lorraine - CNRS, Nancy, France
445000 , about the same number reported in 2015 [1]. Malaria cases were caused by five types of parasite, being Plasmodium falciparum. Most of the treatments used to fight against Plasmodium falciparum include the artemisinin combinatory therapy (ACT). In fact, failures of the ACTs (artesunate/mefloquine and artesunate/amodiaquine) have recently been reported as frequent failures of dihydroartemisinin/piperaquine in parts of Cambodia, and decreasing sensitivity to lumefantrine may further threaten artemether/lumefantrine [2]. Likewise, some strains of Plasmodium falciparum such as FcB1 [2, 3], K1 W2, and TM4 strains are chloroquino-resistant [2-6]. Consequently, the development of new combinations of active compounds becomes crucial to fight against malaria disease.

In the last two decades, some research on xanthone derivatives as anti-malarial agents was published. Winter et al. have identified 2,3,4,5,6-pentahydroxyxanthone (X5) as a potent antimalarial drug with equal activity against multidrug-resistant strains of $P$. falciparum D6 clones [7]. Then, Ignatushchenko et al. have suggested that xanthones act in a unique way to kill Plasmodium through the formation of soluble complexes with heme, therefore inhibiting the process of heme polymerization $[8,9]$. Hay et al. have 
isolated thirteen xanthones from Garcinia vieillardii and have synthetized nine others. They evaluated the antimalarial activity of these xanthones against chloroquino-resistant strains of Plasmodium falciparum FcB1/Colombia. The results showed that the position of the hydroxyl groups appears to be important as indicated by the differences of activities [10]. Concerning the activity of xanthones on P. falciparum K1 strain, Mahabusarakam et al. have shown that the best activity was achieved by addition of alkylamino or hydroxylalkylamino groups to either or both phenolic hydroxyl groups [11]. Upegui et al. have isolated $\alpha$ mangostin and $\delta$-mangostin (1,2,3,6,7-pentahydroxy-4,8 diisoprenylxanthone) from mangosteen husk. They observed that $\alpha$-mangostin was more active against the resistant $P$. falciparum chloroquine-resistant (FCR3) strain (IC50 $=0.2$ $\pm 0.01 \mu \mathrm{M}$ ) than $\delta$-mangostin $(\mathrm{IC} 50=121.2 \pm 1.0 \mu \mathrm{M})$ [12]. In 2016, Auranwiwat et al. isolated and characterized some xanthones; the most active they obtained is 1,6,7-trihydroxy6,6'-dimethyl-2H-pyrano( $\left(2^{\prime}, 3^{\prime}: 3,2\right)$-5-(4-acetoxy-3methylbut-2-enyl)-8-(4-hydroxy-3-methylbut-2-enyl)-xanthone $(\mathrm{IC} 50=6.0 \mu \mathrm{M})$ on strain TM4 of Plasmodium falciparum and bannaxanthones $\mathrm{I}\left(\mathrm{IC}_{50}=3.6 \mu \mathrm{M}\right)$ on $\mathrm{K} 1$ of Plasmodium falciparum [6]. Despite these studies, a model needs to be developed to explain the measured $\mathrm{IC}_{50}$ as well as to predict the biological activity of new compounds. Therefore, it is important to link the antiplasmodial activity of a selection of xanthone derivatives to molecular descriptors in order to propose new efficient compounds. An elegant approach to tackle this issue is to use advanced statistical analysis combined with advanced quantum chemical calculations.

Indeed, the study of the structure-activity relationships (SARs) is useful for the synthesis of new and more active molecules. There are several statistics-based SAR methods for carrying out such task [13-17]. The usual QSAR studies employ equation without any previous conceptual development. Many descriptors use in the equation come from different realms: classical chemistry (solubility, ionization constants, experimental dipole moments, etc.), quantum chemistry (calculated dipole moments, atomic net charges, reactivity indices, etc.), 2D or 3D geometry, graph theory, etc. When several of these indices coming from different realms are mixed in the equation and a solution satisfying all confidence tests is obtained, the problem of the physical interpretation appears due to the empirical character of the equation. In the present study, we use the Klopman-PeradejordiGómez (KPG) approach, which is on the side of the modelbased methods [18]. The equation obtained in this approach is the algebraic form of the hypothesis contained in the model. The KPG approach has proven to be very useful to disclose the relationships between electronic structure and biological activities [19-23]. One of the best proofs that the KPG method is superior to empirical methods is the prediction of the human dose of the DON hallucinogen and the suggestion of a molecule with stronger cannabinoid activity $[22,23]$.

In this paper, we present the results of a quantum-chemical analysis of the relationships between the electronic structure and the antiplasmodial activities of xanthone derivatives against Plasmodium falciparum strain FcB1. From the results obtained, we propose the associated two-dimensional (2D) antiplasmodial partial pharmacophores.

\section{Methods, models, and calculations}

\section{Model and selection of molecules}

\section{Model}

As the Klopman-Peradejordi-Gómez is in use after 25 years, we refer the reader to the references about the model itself [24-30] and its successful applications [22, 29, 31-33]. In summary, a biological activity BA for a given molecule can be represented by the following linear equation:

$$
\begin{aligned}
& \log (B A)=a+b M_{D_{i}}+c \log \left[\sigma_{D_{i}} /(A B C)^{1 / 2}\right]+\sum_{j}\left[e_{j} Q_{j}+f_{j} S_{j}^{E}+s_{j} S_{j}^{N}\right]+\sum_{j} \sum_{m}\left[h_{j}(m) F_{j}(m)+x_{j}(m) S_{j}^{E}(m)\right] \\
& ++\sum_{j} \sum_{m}\left[r_{j}\left(m^{\prime}\right) F_{j}\left(m^{\prime}\right)+t_{j}\left(m^{\prime}\right) S_{j}^{N}\left(m^{\prime}\right)\right]+\sum_{j}\left[g_{j} \mu_{j}+k_{j} \eta_{j}+o_{j} \omega_{j}+z_{j} \varsigma_{j}+w_{j} Q_{j}^{\max }\right]
\end{aligned}
$$

where $a, b, c, e_{\mathrm{j}}, f_{j}, s_{j}, h_{j}(m), x_{j}(m), r_{j}\left(m^{\prime}\right), t_{j}\left(m^{\prime}\right), g_{j}, k_{j}, o_{j}, z_{j}$, and $w_{j}$ are constants, $M_{D_{i}}$ is the drug's mass, $\sigma_{D_{i}}$ is its symmetry number, $A B C$ is the product of the drug's moments of inertia about the three principal axes of rotation, $Q_{\mathrm{j}}$ is the net charge of atom j, $S_{j}^{E}$ and $S_{j}^{N}$ are, respectively, the total atomic electrophilic and nucleophilic superdelocalizabilities of atom $\mathrm{j}, F_{\mathrm{j}, \mathrm{m}}$
$\left(F_{\mathrm{j}, \mathrm{m}^{\prime}}\right)$ is the electron population (Fukui index) of the occupied (vacant) $\mathrm{MO} \mathrm{m}\left(\mathrm{m}^{\prime}\right)$ localized on atom $\mathrm{j}, S_{j}^{E}(m)$ is the atomic electrophilic superdelocalizability of MO m localized on atom $\mathrm{j}, \mu_{j}$ is the local atomic electronic chemical potential of atom $\mathrm{j}, \eta_{j}$ is the local atomic hardness of atom $\mathrm{j}, \omega_{j}$ is the local atomic electrophilicity of atom $\mathrm{j}, \varsigma_{j}$ is the local atomic softness of atom 
$\mathrm{j}, Q_{j}^{\max }$ is the maximum amount of electronic charge that atom $\mathrm{j}$ may accept from another site, and $O_{k}$ is the orientational parameters of the $k$ th substituent.

Throughout this paper, $\mathrm{HOMO}_{\mathrm{j}}{ }^{*}$ refers to the highest occupied molecular orbital localized on atom $\mathrm{j}$ ("local HOMO*”) and $\mathrm{LUMO}_{\mathrm{j}}{ }^{*}$ to the lowest empty MO localized on atom $\mathrm{j}$ ("local LUMO"). The new local atomic reactivity indices of Eq. 1 were derived within the Hartree-Fock-Roothan method and are defined as follows [24]. The descriptors are not normalized because they have a concrete physical meaning and units. Therefore, the coefficients are not normalized. This is necessary for keeping the physics of the equation and also to allow the comparison with other studies carried out with different molecules but owing the same receptors.

The local atomic electronic chemical potential is defined as follows:

$\mu_{i}=\left(\varepsilon_{\text {HOMO }^{*}, i}+\varepsilon_{L U M O}^{*}, i\right) / 2$

and represents the measure of the propensity of atom i to gain or lose electrons.

The local atomic hardness is defined as follows:

$\eta_{i}=\left(\varepsilon_{H O M O *, i}-\varepsilon_{L U M O *, i}\right)$

This index measures the resistance of atom $i$ to exchange electrons with a site.

The local electrophilic superdelocalizability of the HOMO* of atom $\mathrm{i}$ :

$S_{i}^{E^{*}}=\frac{F_{i, H O M O^{*}}}{\varepsilon_{\text {HОMO* }}}$

The physical meaning of this index is the electron-donating capacity of atom $i$ at occupied $\mathrm{MO}$ m.

The local nucleophilic superdelocalizability of the LUMO* of atom i:

$S_{i}^{N *}=\frac{F_{i, L U M O^{*}}}{\varepsilon_{L U M O^{*}}}$

It is a measure of the electron-accepting capacity of atom $\mathrm{i}$ at empty MO m'.

The local atomic softness of atom $i$ is defined as:

$s_{i}=\frac{1}{\eta_{i}}$

This index is the inverse of the hardness, so it measures the facility of atom i to exchange electrons with the site.

Local atomic electrophilicity of atom $i$ is defined as:

$\omega_{i}=\frac{\mu_{i}^{2}}{2 \eta_{i}}$
This index measures the propensity of atom $i$ to receive extra electronic charge together with its resistance to exchange charge with a site.

The maximal amount of which charge atom i may receive is defined as:

$Q_{i}^{\max }=-\frac{\mu_{i}}{\eta_{i}}$

From a conceptual perspective, the work presented here is a test of the hypothesis stating that the KPG model can provide a quantitative and formal relationship between the molecular structure and any biological activity. Nowadays, the KPG model produced excellent results in all its applications [21, 29, 32, 33].

\section{Selection of molecules}

Molecules were selected from a set reported in the literature [10]. The molecules are shown in Fig. 1 and Table 1. Figure 2 shows the numbering of atoms used in the linear multiple regression analysis (LMRA). The experimental data employed in this study are in vitro antiplasmodial activity on Plasmodium falciparum strain FcB1/Colombia quantified as $\mathrm{IC}_{50}[10]$.

\section{Calculations}

The electronic structure of the molecules was obtained within the density functional theory (DFT) at the B3LYP/6-31G(d,p) level of theory with full geometry optimization. The Gaussian collection of programs was employed [34]. The local atomic reactivity indices were calculated from the single point log file with the D-Cent-QSAR software [35] with correction of the anomalous electron populations that sometimes are produced by the Mulliken population analysis [36]. All electron populations smaller than or equal to $0.01 \mathrm{e}$ were considered as zero [36]. We employed the common skeleton hypothesis defined as a particular set of atoms, common to all molecules analyzed, that accounts for nearly all the biological activity. The variation of the values of a set of local atomic reactivity indices of one or more atoms belonging to this skeleton gives an account of the variation of the antiplasmodial activity

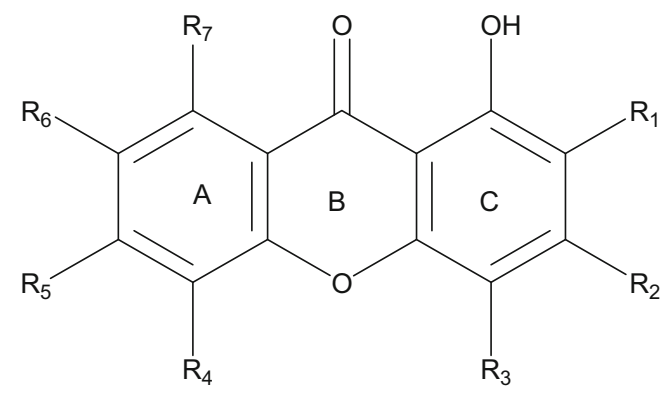

Fig. 1 Structure of xanthone derivatives 
Table 1 Selected molecules and their antiplasmodial activities

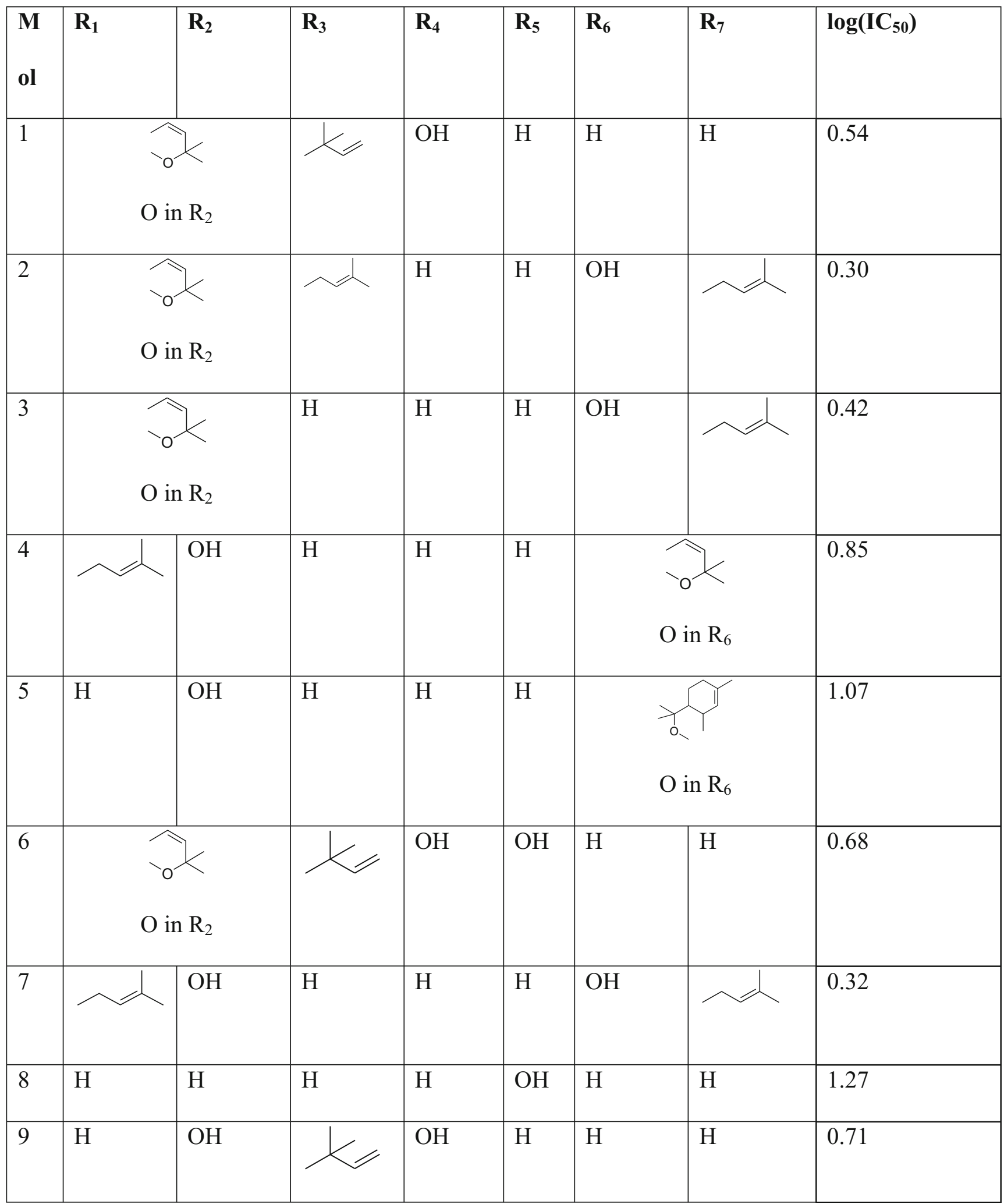


Table 1 (continued)

\begin{tabular}{|c|c|c|c|c|c|c|c|c|}
\hline M & $\mathbf{R}_{1}$ & $\mathbf{R}_{2}$ & $\mathbf{R}_{3}$ & $\mathbf{R}_{4}$ & $\mathbf{R}_{5}$ & $\mathbf{R}_{6}$ & $\mathbf{R}_{7}$ & $\log \left(\mathrm{IC}_{50}\right)$ \\
\hline 10 & $\mathrm{H}$ & $\mathrm{OMe}$ & & $\mathrm{OH}$ & $\mathrm{OH}$ & $\mathrm{H}$ & $\mathrm{H}$ & 0.97 \\
\hline 11 & $\mathrm{H}$ & $\mathrm{OH}$ & & $\mathrm{OH}$ & $\mathrm{OH}$ & $\mathrm{H}$ & $\mathrm{H}$ & 0.85 \\
\hline 12 & $\mathrm{H}$ & & & $\mathrm{OH}$ & $\mathrm{OH}$ & $\mathrm{H}$ & $\mathrm{H}$ & 1.03 \\
\hline 13 & $\mathrm{H}$ & $\mathrm{H}$ & $\mathrm{OH}$ & $\mathrm{OH}$ & $\mathrm{H}$ & $\mathrm{H}$ & $\mathrm{H}$ & 1.16 \\
\hline 14 & $\mathrm{H}$ & $\mathrm{OH}$ & $\mathrm{H}$ & $\mathrm{OH}$ & $\mathrm{H}$ & $\mathrm{H}$ & $\mathrm{H}$ & 2.01 \\
\hline 15 & & $\mathrm{OH}$ & $\mathrm{H}$ & $\mathrm{OH}$ & $\mathrm{H}$ & $\mathrm{H}$ & $\mathrm{H}$ & 1.20 \\
\hline 16 & & $\mathrm{OH}$ & & $\mathrm{OH}$ & $\mathrm{H}$ & $\mathrm{H}$ & $\mathrm{H}$ & 0.68 \\
\hline 17 & $\mathrm{H}$ & $\mathrm{OH}$ & & $\mathrm{OH}$ & $\mathrm{H}$ & $\mathrm{H}$ & $\mathrm{H}$ & 1.12 \\
\hline 18 & & $\mathrm{OH}$ & $\mathrm{H}$ & $\mathrm{OH}$ & $\mathrm{H}$ & $\mathrm{H}$ & $\mathrm{H}$ & 1.05 \\
\hline 19 & $\mathrm{H}$ & $\mathrm{OH}$ & & $\mathrm{OH}$ & $\mathrm{H}$ & $\mathrm{H}$ & $\mathrm{H}$ & 1.30 \\
\hline 20 & $\mathrm{H}$ & & $\mathrm{R}_{2}$ & $\mathrm{OH}$ & $\mathrm{H}$ & $\mathrm{H}$ & $\mathrm{H}$ & 0.65 \\
\hline 21 & & $\mathrm{OH}$ & & $\mathrm{OH}$ & $\mathrm{H}$ & $\mathrm{H}$ & $\mathrm{H}$ & 1.56 \\
\hline 22 & & & & $\mathrm{OH}$ & $\mathrm{H}$ & $\mathrm{H}$ & $\mathrm{H}$ & 1.14 \\
\hline
\end{tabular}


throughout the series analyzed. The common skeleton numbering is shown in Fig. 2.

We made use of linear multiple regression analysis (LMRA) using the Statistica software [37] to determine which atoms are directly involved in the variation of the biological activity. We built a matrix containing the dependent variable $\left(\log \left(\mathrm{IC}_{50}\right)\right)$, and the local atomic reactivity indices of all atoms of the common skeleton as independent variables.

\section{Results}

The best statistically significant equation obtained is the following:

$$
\begin{aligned}
\log \left(\mathrm{IC}_{50}\right)= & 9.45-1.97 \mu_{6}-1.84 S_{15}^{N}(L U M O)^{*}-17.61 S_{16}^{E}(\text { HOMO })^{*} \\
& -20.18 F_{16}(\text { HOMO }-2)^{*}-50.56 Q_{16}-0.81 F_{5}(\text { LUMO }+1)^{*} \\
& +0.14 S_{8}^{E}(\text { HOMO }-2)^{*}+0.07 S_{16}^{N}(L U M O+1)^{*}
\end{aligned}
$$

with $n=22, R=0.99, R^{2}=0.98$, adj- $R^{2}=0.97, F(8.13)=81.33$, $p<0.000001$, and a standard error estimate of 0.07 . No outliers were detected and no residual fall outside the $\pm 2 \sigma$ limits. Here, $\mu_{6}$ is the local atomic electronic chemical potential of atom $6, S_{15}^{N}$ $(L U M O)^{*}$ is the atomic nucleophilic superdelocalizability of the lowest empty MO localized on atom $15, S_{16}^{E}(H O M O)^{*}$ is the atomic electrophilic superdelocalizability of highest occupied MO localized on atom 16, $F_{16}(H O M O-2)^{*}$ is the Fukui index of the third highest occupied MO localized on atom $16, Q_{16}$ is the net charge of atom $16, F_{5}(L U M O+1)^{*}$ is the Fukui index of the vacant second lowest empty MO localized on atom $5, S_{8}^{E}$ $(H O M O-2)^{*}$ is the atomic electrophilic superdelocalizability of the third highest occupied MO localized on atom 8 , and $S_{16}^{N}$ $(L U M O+1)^{*}$ is the atomic nucleophilic superdelocalizability of (LUMO+1)* on atom 16.

The KPG method uses a model-based equation and must have a solution by definition. We use statistics to search the best equation and not to check if there is one. KPG method has not the

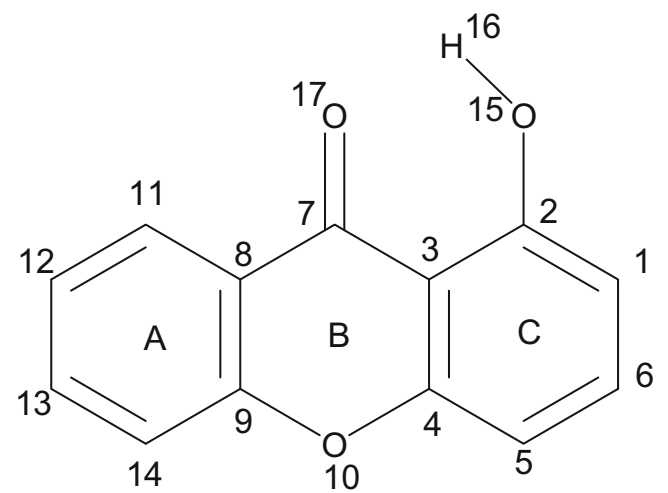

Fig. 2 Common skeleton of xanthone derivatives obligation to perform the external and internal validations because of its mathematical formal structure. Table 2 shows the beta coefficients and the $t$ test results for the significance of coefficients of Eq. 9. Table 3 shows the squared correlation coefficients for the variables appearing in Eq. 9.

Table 3 shows that the highest internal correlation is $r^{2}$ $\left(S_{15}^{N}(L U M O)^{*}, \mu_{6}\right)=0.26$. Figure 3 shows the plot of observed values vs. calculated values of $\log \left(\mathrm{IC}_{50}\right)$. The associated statistical parameters of Eq. 9 show that this equation is statistically significant and that the variation of the numerical values of eight LARIs explains about $95 \%$ of the variation of the biological activity.

To be able to suggest the type of molecular interactions that involve atoms appearing in Eq. 9, we determine the nature of their three highest occupied and three lowest empty local molecular orbitals. Table 4 shows the local molecular orbitals of atoms 5, 6, 15, and 16 (see Fig. 3). Nomenclature: Molecule $(\mathrm{HOMO}) /(\mathrm{HOMO}-2) *(\mathrm{HOMO}-1) *(\mathrm{HOMO}) *$ - (LUMO)* $(\mathrm{LUMO}+1)^{*}(\mathrm{LUMO}+2)^{*}$. The number corresponding of the HOMO of each molecule is in bracket, and for each atom, we have the third local HOMO* and the third local LUMO*.

The molecular orbitals of molecule 2 (one of the most active) are represented in Fig. 4. Note that this MO is localized only on some atoms.

\section{Discussion}

The beta values (Table 3 ) shows that the importance of variables decreases in the following order: $\mu_{6}>S_{15}^{N}(L U M O)^{*}$ $\approx S_{16}^{E}(H O M O)^{*} \approx F_{16}(H O M O-2)^{*}>Q_{16}>F_{5}(L U M O+$ $1)^{*}>S_{8}^{E}(H O M O-2)^{*} \approx S_{16}^{N}(L U M O+1)^{*}$. Table 3 indicates that $S_{8}^{E}(H O M O-2)^{*}$ and $S_{16}^{N}(L U M O+1)^{*}$ have a low significance since the associated $p$ values are higher than 0.005 . Therefore, we will discuss only the six others indices. The process seems to be orbital and charge-controlled because seven

Table 2 Beta coefficients and $t$ test for significance of coefficients in Eq. 9

\begin{tabular}{llll}
\hline Variable & Beta & $t(10)$ & $p$ level \\
\hline$\mu_{6}$ & -0.90 & -18.52 & $<0.000000$ \\
$S_{15}^{N}(\text { LUMO })^{*}$ & -0.42 & -7.88 & $<0.000003$ \\
$S_{16}^{E}(H O M O)^{*}$ & -0.41 & -9.43 & $<0.000000$ \\
$F_{16}(H O M O-2)^{*}$ & -0.40 & -7.20 & $<0.000007$ \\
$Q_{16}$ & -0.33 & -6.75 & $<0.000014$ \\
$F_{5}(L U M O+1)^{*}$ & -0.19 & -3.68 & $<0.003$ \\
$S_{8}^{E}(H O M O-2)^{*}$ & 0.14 & 3.05 & $<0.009$ \\
$S_{16}^{N}(L U M O+1)^{*}$ & 0.12 & 3.02 & $<0.010$ \\
\hline
\end{tabular}


Table 3 Squared correlation coefficients for the variables appearing in Eq. 9

\begin{tabular}{|c|c|c|c|c|c|c|c|}
\hline & $\mu_{6}$ & $S_{15}^{N}(L U M O)^{*}$ & $S_{16}^{E}(H O M O)^{*}$ & $F_{16}(H O M O-2)^{*}$ & $Q_{16}$ & $F_{5}(L U M O+1)^{*}$ & $S_{8}^{E}(H O M O-2)^{*}$ \\
\hline$S_{15}^{N}(L U M O)^{*}$ & 0.26 & & & & & & \\
\hline$S_{16}^{E}(H O M O)^{*}$ & 0.01 & 0.08 & & & & & \\
\hline$F_{16}(H O M O-2)^{*}$ & 0.01 & 0.02 & 0.02 & & & & \\
\hline$Q_{16}$ & 0.01 & 0.05 & 0.00 & 0.17 & & & \\
\hline$F_{5}(L U M O+1)^{*}$ & 0.00 & 0.07 & 0.08 & 0.23 & 0.01 & & \\
\hline$S_{8}^{E}(H O M O-2)^{*}$ & 0.04 & 0.10 & 0.01 & 0.07 & 0.19 & 0.01 & \\
\hline$S_{16}^{N}(L U M O+1)^{*}$ & 0.01 & 0.00 & 0.00 & 0.08 & 0.00 & 0.00 & 0.01 \\
\hline
\end{tabular}

indices depend on the electron population or/and the energies of the MOs and one depend on the net charge of atom 16.

For the discussion, we shall use the variable by variable $(\mathrm{VbV})$ analysis. The local electronic chemical potentials are negative; the Fukui indices are always positive; the electrophilic superdelocalizabilities, total and partial, are negative; nucleophilic superdelocalizability is positive in general cases; and the charge of atom 16 is positive. So the $\mathrm{VbV}$ analysis shows that a good activity should be associated with low negative numerical values of $\mu_{6}$ and $S_{16}^{E}(H O M O)^{*}$, high positive numerical values of $S_{15}^{N}(L U M O)^{*}, F_{16}(H O M O-2)^{*}, Q_{16}$, and $F_{5}(L U M O+1)^{*}$.

Let us now discuss these findings at the molecular scale.

Indeed, atom 6 is a carbon atom of the ring $\mathrm{C}$ (Fig. 2). The small negative value of $\mu_{6}$ should be obtained by making less negative the $\mathrm{HOMO}^{*}$ energy, making this atom a good electron donor. In Table 4, we observe two cases about the position of HOMO* and LUMO*. In the first case, the local $\mathrm{HOMO}^{*}$ of atom 6 does not coincide with the molecular HOMO and the LUMO* coincides with the molecular LUMO (Table 4). In the second case, the local HOMO* of atom 6 coincides with the molecular
HOMO and the LUMO* coincides with the molecular LUMO. In the first case, we have to make the HOMO* more reactive; in the second case, the LUMO* should be less reactive. Therefore, atom 6 seems to interact with an electron deficient center of a probable $\pi$ nature.

Atom 15 is an oxygen atom of the hydroxyl group on the ring $\mathrm{C}$ (Fig. 2). The high positive value of $S_{15}^{N}(L U M O)^{*}$ should be obtained whether by raising the Fukui index of $\mathrm{LUMO}_{15}$ * or by lowering the $\mathrm{LUMO}_{15}$ * energy. In both cases, the $\mathrm{LUMO}_{15} *$ will be more reactive, so atom 15 interacts with an electron-rich center through its $\pi$ empty orbitals.

Atom 16 is a hydrogen atom of the hydroxyl group on the ring $\mathrm{C}$ (Fig. 2). A good activity is associated with the low negative value of $S_{16}^{E}(H O M O)^{*}$ so the corresponding orbital should be less reactive and should be an inner MO. It is the case in all the molecules because $\mathrm{HOMO}_{16}{ }^{*}$ is energetically far from the molecular HOMO (Table 4). Atom 16 should interact with an electron-rich center. So atom 16 could participate to H-bond. This fact satisfies to the high value of $Q_{16}$. A high value of $F_{16}(\mathrm{HOMO}-2)^{*}$ does not affect the value of $S_{16}^{E}(H O M O)^{*}$ and it would be obtained easily because we have the inner MO.
Fig. 3 Plot of predicted vs. observed $\log \left(\mathrm{IC}_{50}\right)$ values. Dashed lines denote the $95 \%$ confidence interval

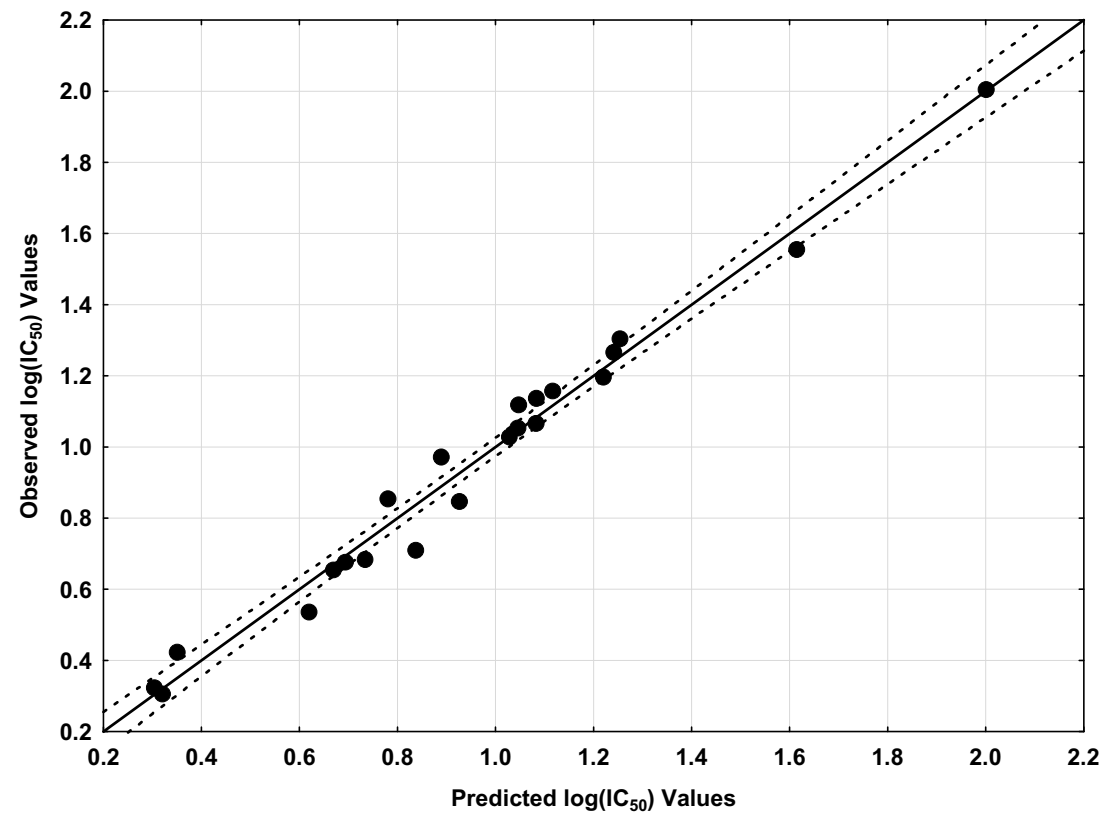


Table 4 Local molecular orbitals of atoms 5, 6, 15, and 16

\begin{tabular}{lllll}
\hline Mol. & Atom $5(\mathrm{C})$ & Atom $6(\mathrm{C})$ & Atom $15(\mathrm{O})$ & Atom $16(\mathrm{H})$ \\
\hline $1(100)$ & $96 \pi 99 \pi 100 \pi-101 \pi 102 \pi 103 \pi$ & $93 \pi 98 \pi 99 \pi-101 \pi 102 \pi 103 \pi$ & $98 \pi 99 \pi 100 \pi-101 \pi 102 \pi 103 \pi$ & $80 \sigma 82 \sigma 85 \sigma-102 \sigma 103 \sigma 105 \sigma$ \\
$2(119)$ & $116 \pi 117 \pi 119 \pi-120 \pi 121 \pi 122 \pi$ & $111 \pi 116 \pi 117 \pi-120 \pi 121 \pi 122 \pi$ & $117 \pi 118 \pi 119 \pi-120 \pi 121 \pi 122 \pi$ & $94 \sigma 100 \sigma 102 \sigma-121 \sigma 123 \sigma 124 \sigma$ \\
$3(100)$ & $98 \pi 99 \pi 100 \pi-102 \pi 103 \pi 104 \pi$ & $98 \pi 99 \pi 100 \pi-101 \pi 102 \pi 103 \pi$ & $97 \pi 98 \pi 100 \pi-101 \pi 102 \pi 103 \pi$ & $85 \sigma 88 \sigma 96 \sigma-113 \sigma 114 \sigma 115 \sigma$ \\
$4(100)$ & $98 \pi 99 \pi 100 \pi-101 \pi 102 \pi 103 \pi$ & $94 \pi 97 \pi 99 \pi-101 \pi 102 \pi 103 \pi$ & $98 \pi 99 \pi 100 \pi-101 \pi 102 \pi 104 \pi$ & $87 \sigma 89 \sigma 95 \sigma-104 \sigma 108 \sigma 109 \sigma$ \\
$5(100)$ & $97 \pi 99 \pi 100 \pi-101 \pi 102 \pi 103 \pi$ & $96 \pi 97 \pi 99 \pi-101 \pi 102 \pi 103 \pi$ & $98 \pi 99 \pi 100 \pi-101 \pi 103 \pi 104 \pi$ & $80 \sigma 83 \sigma 84 \sigma-106 \sigma 107 \sigma 110 \sigma$ \\
$6(104)$ & $102 \pi 103 \pi 104 \pi-106 \pi 108 \pi 109 \pi$ & $102 \pi 103 \pi 104 \pi-105 \pi 106 \pi 107 \pi$ & $102 \pi 103 \pi 104 \pi-105 \pi 106 \pi 108 \pi$ & $87 \sigma 90 \sigma 99 \sigma-118 \sigma 121 \sigma 123 \sigma$ \\
$7(101)$ & $99 \pi 100 \pi 101 \pi-102 \pi 103 \pi 104 \pi$ & $94 \pi 97 \pi 98 \pi-102 \pi 103 \pi 104 \pi$ & $98 \pi 99 \pi 101 \pi-102 \pi 104 \pi 105 \pi$ & $86 \sigma 95 \sigma 96 \sigma-105 \sigma 108 \sigma 110 \sigma$ \\
$8(59)$ & $56 \pi 57 \pi 59 \pi-60 \pi 61 \pi 62 \pi$ & $55 \pi 56 \pi 59 \pi-60 \pi 61 \pi 62 \pi$ & $56 \pi 57 \pi 59 \pi-60 \pi 63 \pi 70 \pi$ & $46 \sigma 50 \sigma 51 \sigma-65 \sigma 67 \sigma 68 \sigma$ \\
$9(82)$ & $79 \pi 81 \pi 82 \pi-83 \pi 84 \pi 85 \pi$ & $79 \pi 81 \pi 82 \pi-83 \pi 84 \pi 85 \pi$ & $80 \pi 81 \pi 82 \pi-83 \pi 86 \pi 87 \pi$ & $66 \sigma 69 \sigma 70 \sigma-89 \sigma 90 \sigma 92 \sigma$ \\
$10(90)$ & $88 \pi 89 \pi 90 \pi-91 \pi 92 \pi 93 \pi$ & $87 \pi 89 \pi 90 \pi-91 \pi 92 \pi 93 \pi$ & $88 \pi 89 \pi 90 \pi-91 \pi 93 \pi 94 \pi$ & $71 \sigma 74 \sigma 79 \sigma-97 \sigma 98 \sigma 99 \sigma$ \\
$11(86)$ & $84 \pi 85 \pi 86 \pi-87 \pi 88 \pi 89 \pi$ & $83 \pi 85 \pi 86 \pi-87 \pi 88 \pi 89 \pi$ & $84 \pi 85 \pi 861 p-87 \pi 90 \pi 96 \pi$ & $68 \sigma 71 \sigma 75 \sigma-91 \sigma 93 \sigma 94 \sigma$ \\
$12(86)$ & $82 \pi 85 \pi 86 \pi-87 \pi 88 \pi 89 \pi$ & $84 \pi 85 \pi 86 \pi-87 \pi 88 \pi 89 \pi$ & $83 \pi 84 \pi 86 \pi-87 \pi 90 \pi 97 \pi$ & $71 \sigma 73 \sigma 74 \sigma-92 \sigma 93 \sigma 96 \sigma$ \\
$13(63)$ & $58 \pi 62 \pi 63 \pi-64 \pi 65 \pi 66 \pi$ & $60 \pi 62 \pi 63 \pi-64 \pi 65 \pi 66 \pi$ & $60 \pi 62 \pi 63 \pi-64 \pi 67 \pi 74 \pi$ & $51 \sigma 54 \sigma 55 \sigma-69 \sigma 70 \sigma 72 \sigma$ \\
$14(63)$ & $59 \pi 62 \pi 63 \pi-64 \pi 65 \pi 66 \pi$ & $59 \pi 60 \pi 62 \pi-64 \pi 65 \pi 66 \pi$ & $61 \pi 62 \pi 63 \pi-64 \pi 66 \pi 67 \pi$ & $51 \sigma 52 \sigma 54 \sigma-68 \sigma 69 \sigma 70 \sigma$ \\
$15(82)$ & $80 \pi 81 \pi 82 \pi-83 \pi 84 \pi 86 \pi$ & $79 \pi 80 \pi 81 \pi-83 \pi 84 \pi 86 \pi$ & $80 \pi 81 \pi 82 \pi-83 \pi 85 \pi 86 \pi$ & $71 \sigma 7477 \sigma-85 \sigma 89 \sigma 90 \sigma$ \\
$16(101)$ & $95 \pi 100 \pi 101 \pi-102 \pi 103 \pi 104 \pi$ & $97 \pi 98 \pi 100 \pi-102 \pi 103 \pi 104 \pi$ & $99 \pi 100 \pi 101 \pi-102 \pi 104 \pi 105 \pi$ & $92 \sigma 96 \sigma 97 \sigma-105 \sigma 109 \sigma 110 \sigma$ \\
$17(82)$ & $80 \pi 81 \pi 82 \pi-83 \pi 84 \pi 85 \pi$ & $80 \pi 81 \pi 82 \pi-83 \pi 84 \pi 85 \pi$ & $80 \pi 81 \pi 82 \pi-83 \pi 85 \pi 86 \pi$ & $64 \sigma 65 \sigma 70 \sigma-88 \sigma 89 \sigma 90 \sigma$ \\
$18(86)$ & $84 \pi 85 \pi 86 \pi-87 \pi 88 \pi 90 \pi$ & $83 \pi 84 \pi 85 \pi-87 \pi 88 \pi 90 \pi$ & $81 \pi 85 \pi 86 \pi-87 \pi 89 \pi 90 \pi$ & $75 \sigma 77 \sigma 78 \sigma-89 \sigma 94 \sigma 98 \sigma$ \\
$19(86)$ & $83 \pi 85 \pi 86 \pi-87 \pi 88 \pi 89 \pi$ & $83 \pi 85 \pi 86 \pi-87 \pi 88 \pi 89 \pi$ & $84 \pi 85 \pi 86 \pi-87 \pi 89 \pi 90 \pi$ & $71 \sigma 73 \sigma 74 \sigma-94 \sigma 95 \sigma 96 \sigma$ \\
$20(81)$ & $76 \pi 80 \pi 81 \pi-82 \pi 83 \pi 86 \pi$ & $78 \pi 80 \pi 81 \pi-82 \pi 83 \pi 84 \pi$ & $78 \pi 79 \pi 81 \pi-82 \pi 83 \pi 84 \pi$ & $66 \sigma 67 \sigma 69 \sigma-87 \sigma 88 \sigma 89 \sigma$ \\
$21(109)$ & $107 \pi 108 \pi 109 \pi-111 \pi 112 \pi 113 \pi$ & $106 \pi 107 \pi 108 \pi-110 \pi 111 \pi 112 \pi$ & $104 \pi 108 \pi 109 \pi-110 \pi 112 \pi 113 \pi$ & $88 \sigma 89 \sigma 103 \sigma-121 \sigma 123 \sigma 124 \sigma$ \\
$22(104)$ & $98 \pi 103 \pi 104 \pi-105 \pi 106 \pi 110 \pi$ & $101 \pi 103 \pi 104 \pi-105 \pi 106 \pi 107 \pi$ & $101 \pi 102 \pi 104 \pi-105 \pi 106 \pi 107 \pi$ & $90 \sigma 93 \sigma 94 \sigma-110 \sigma 111 \sigma 113 \sigma$ \\
\hline & & & &
\end{tabular}

Atom 5 is a carbon of ring $\mathrm{C}$ (Fig. 2). The fact that $(\mathrm{LUMO}+1)_{5} *$ appears in the equation indicates that $\mathrm{LUMO}_{5} *$ is also participating to the process. All the three

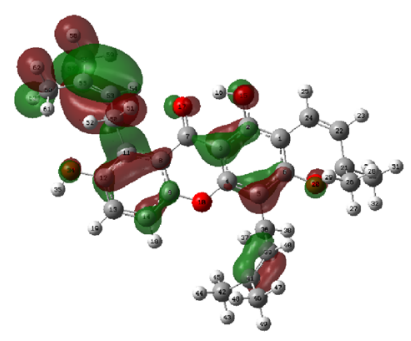

HOMO-2

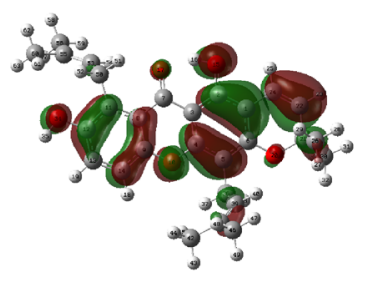

HOMO

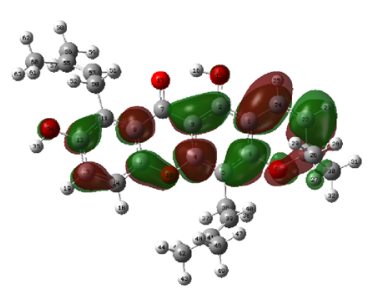

$\mathrm{LUMO}+1$

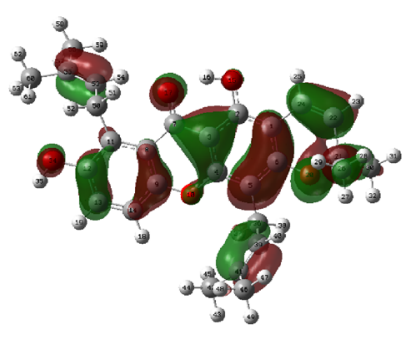

HOMO-1
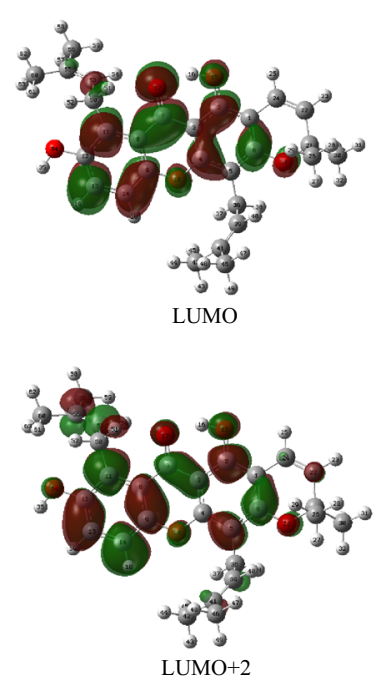

Fig. 4 Molecular orbitals of molecule 2 lowest vacant orbitals localized on atom 5 are $\pi$ nature (Table 4). The high value of $F_{5}(L U M O+1)^{*}$ indicates that atom 5 should interact with electron-rich center; this interaction could be $\pi-\pi$ or $\pi-\sigma$ kind.

All these suggestions are presented in the partial 2D inhibition pharmacophore of Fig. 5 .

\section{Conclusions}

We obtained a statistically significant relationship between the variation of the antiplasmodial activities of some xanthones derivatives and the variation of the numerical values of a set of local atomic reactivity indices. This allowed us to build the

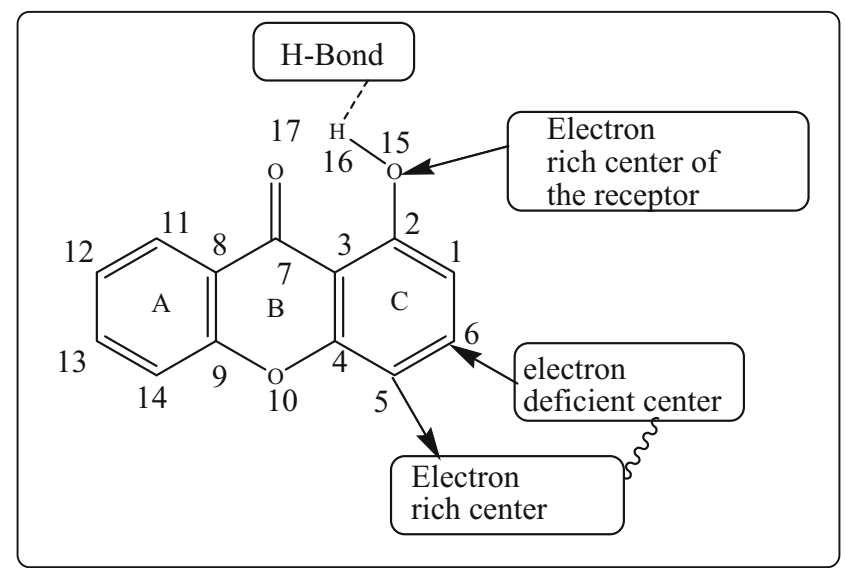

Fig. 5 Partial 2D pharmacophore for the antiplasmodial activities of xanthone derivatives on Plasmodium falciparum strain FcB1 
associated pharmacophore that should serve as a starting point for chemical modifications producing more active compounds. The 2D-pharmacophore shows that the substitution on one ring can improve the antiplasmodial activity, and the presence of hydroxyl group near the carbonyl group plays an important function.

\section{Compliance with ethical standards}

Conflict of interest The authors declare that they have no conflict of interest.

\section{References}

1. World Health Organization and Global Malaria Programme World malaria report 2017 (2017) https://www.who.int/malaria/ publications/worldmalaria-report-2017/en/. Accessed 29 Nov 2017

2. Rosenthal PJ, Rathod PK, Ndiaye D, Mharakurwa S, Cui L (2015) Antimalarial drug resistance: literature review and activities and findings of the ICEMR network. Am. J. Trop. Med. Hyg. 93:57-68

3. Menard D, Dondorp A (2017) Antimalarial drug resistance: a threat to malaria elimination. Cold Spring Harb. Perspect. Med. 7: a025619

4. Boudhar A, Ng XW, Loh CY, Chia WN, Tan ZM, Nosten F, Dymock BW, Tan KSW (2016) Overcoming chloroquine resistance in malaria: design, synthesis and structure-activity relationships of novel chemoreversal agents. Eur. J. Med. Chem. 119:231249

5. Kalaria PN, Karad SC, Raval DK (2018) A review on diverse heterocyclic compounds as the privileged scaffolds in antimalarial drug discovery. Eur. J. Med. Chem. 158:917-936

6. Auranwiwat C, Laphookhieo S, Rattanajak R, Kamchonwongpaisan S, Pyne SG, Ritthiwigrom T (2016) Antimalarial polyoxygenated and prenylated xanthones from the leaves and branches of Garcinia mckeaniana. Tetrahedron 72:6837-6842

7. Winter RW, Cornell KA, Johnson LL, Ignatushchenko M, Hinrichs DJ, Riscoe MK (1996) Potentiation of the antimalarial agent rufigallol. Antimicrob. Agents Chemother. 40:1408-1411

8. Ignatushchenko MV, Winter RW, Bächinger HP, Hinrichs DJ, Riscoe MK (1997) Xanthones as antimalarial agents; studies of a possible mode of action. FEBS Lett. 409:67-73

9. Ignatushchenko MV, Winter RW, Riscoe M (2000) Xanthones as antimalarial agents: stage specificity. Am. J. Trop. Med. Hyg. 62: $77-81$

10. Hay A-E, Hélesbeux J-J, Duval O, Labaïed M, Grellier P, Richomme P (2004) Antimalarial xanthones from Calophyllum caledonicum and Garcinia vieillardii. Life Sci. 75:3077-3085

11. Mahabusarakam W, Kuaha K, Wilairat P, Taylor W (2006) Prenylated xanthones as potential antiplasmodial substances. Planta Med. 72:912-916

12. Upegui Y, Robledo SM, Gil Romero JF, Quiñones W, Archbold R, Torres F, Escobar G, Nariño B, Echeverri F (2015) In vivo antimalarial activity of $\alpha$-mangostin and the new xanthone $\delta$-mangostin: antimalarial activity of a new xanthone. Phytother. Res. 29:11951201

13. Noguera GJ, Fabian LE, Lombardo E, Finkielsztein L (2015) QSAR study and conformational analysis of 4-arylthiazolylhydrazones derived from 1-indanones with anti-Trypanosoma cruzi activity. Eur. J. Pharm. Sci. 78:190-197
14. Shayanfar A, Shayanfar S (2014) Is regression through origin useful in external validation of QSAR models? Eur. J. Pharm. Sci. 59: $31-35$

15. Shibi IG, Aswathy L, Jisha RS, Masand VH, Divyachandran A, Gajbhiye JM (2015) Molecular docking and QSAR analyses for understanding the antimalarial activity of some 7-substituted-4aminoquinoline derivatives. Eur. J. Pharm. Sci. 77:9-23

16. Cheng Y, Luo F, Zeng Z, Wen L, Xiao Z, Bu H, Lv F, Xu Z, Lin Q (2015) DFT-based quantitative structure-activity relationship studies for antioxidant peptides. Struct. Chem. 26: 739-747

17. Toropov AA, Toropova AP, Benfenati E, Gini G, Fanelli R (2013) The definition of the molecular structure for potential anti-malaria agents by the Monte Carlo method. Struct. Chem. 24:1369-1381

18. Martin YC (1978) Quantitative drug design: a critical introduction Print book: English. Marcel Dekker, New York

19. Gomez-Jeria JS, Orellana Í (2016) A theoretical analysis of the inhibition of the VEGFR-2 vascular endothelial growth factor and the anti-proliferative activity against the HepG2 hepatocellular carcinoma cell line by a series of 1-(4-((2oxoindolin-3-ylidene)amino)phenyl)-3-arylureas. Pharma Chem. 8:476-487

20. Gomez-Jeria JS, Valdebenito-Gamboa J (2015) A quantumchemical analysis of the antiproliferative activity of N-3benzimidazolephenylbisamide derivatives against MGC803, HT29, MKN45 and SW620 cancer cell lines. Pharma Chem. 7: $103-121$

21. Robles-Navarro A, Gómez Jeria J (2016) A quantum-chemical analysis of the relationships between electronic structure and cytotoxicity, GyrB inhibition, DNA supercoiling inhibition and antitubercular activity of a series of quinoline-aminopiperidine hybrid analogues. Pharma Chem. 8:417-440

22. Gomez-Jeria JS, Cassels BK, Saavedra-Aguilar JC (1987) A quantum-chemical and experimental study of the hallucinogen ( \pm )-1-(2,5-dimethoxy-4-nitrophenyl)-2-aminopropane (DON). Eur. J. Med. Chem. 22:433-437

23. GóMez-Jeria JS, Soto-Morales F, Rivas J, Sotomayor A (2008) A theoretical structure-affinity relationship study of some cannabinoid DERIVATIVES. J. Chil. Chem. Soc. 53

24. Gómez Jeria JS (2013) A new set of local reactivity indices within the Hartree-Fock-Roothaan and density functional theory frameworks. Can. Chem. Trans. 1:25-55

25. Gómez Jeria JS (2013) Elements of molecular electronic pharmacology1st edn. Ediciones Sokar, Santiago de Chile

26. Gómez Jeria JS (1982) Calculation of the nucleophilic superdelocalizability by the CNDO/2 method. J. Pharm. Sci. 71: $1423-1424$

27. Gómez Jeria JS (1982) La Pharmacologie Quantique. Boll. Chim. Farm. 121:619-625

28. Gomez-Jeria JS (1983) On some problems in quantum pharmacology I. The partition functions. Int. J. Quantum Chem. 23:19691972

29. Gómez Jeria JS, Flores-Catalán M (2013) Quantum-chemical modeling of the relationships between molecular structure and in vitro multi-step, multimechanistic drug effects. HIV-1 replication inhibition and inhibition of cell proliferation as examples. Can. Chem. Trans. 1:215-237

30. Gómez-Jeria JS (1989) Modeling the drug-receptor interaction in quantum pharmacology. In: Maruani J (ed) Molecules in physics, chemistry, and biology. Springer Netherlands, Dordrecht, pp 215231

31. Gomez-Jeria JS, Sotomayor P (1988) Quantum chemical study of electronic structure and receptor binding in opiates. J. Mol. Struct. THEOCHEM 166:493-498 
32. Kpotin G, Atohoun SYG, Kuevi AU, Kpota-Hounguè A, Mensah J-B, Gómez Jeria JS (2016) A quantum-chemical study of the relationships between electronic structure and trypanocidal activity against Trypanosoma brucei brucei of a series of thiosemicarbazone derivatives. Pharm. Lett. 8:215-222

33. Gómez-Jeria JS, Cornejo-Martínez P (2016) A DFT study of the inhibition of human phosphodiesterases PDE3A and PDE3B by a group of 2-(4-(1H-tetrazol-5-yl)-1H-pyrazol-1-yl)- 4-(4phenyl)thiazole derivatives. Pharma Chem. 8:329-337

34. Frisch MJ, Trucks GW, Schlegel HB, Scuseria GE, Robb MA, Cheeseman JR, Montgomery JA Jr, Vreven T, Kudin KN, Burant JC, Millam JM, Iyengar SS, Tomasi J, Barone V, Mennucci B, Cossi M, Scalmani G, Rega N, Petersson GA, Nakatsuji H, Hada M, Ehara M, Toyota K, Fukuda R, Hasegawa J, Ishida M, Nakajima T, Honda Y, Kitao O, Nakai H, Klene M, Li X, Knox JE, Hratchian HP, Cross JB, Adamo C, Jaramillo J, Gomperts R, Stratmann RE, Yazyev O, Austin AJ, Cammi R, Pomelli C, Ochterski JW, Ayala PY, Morokuma K, Voth GA, Salvador P, Dannenberg JJ, Zakrzewski VG, Dapprich S, Daniels AD, Strain MC, Farkas O, Malick DK, Rabuck AD, Raghavachari K, Foresman JB, Ortiz JV, Cui Q, Baboul AG, Clifford S, Cioslowski J, Stefanov BB, Liu G,
Liashenko A, Piskorz P, Komaromi I, Martin RL, Fox DJ, Keith T, Al-Laham MA, Peng CY, Nanayakkara A, Challacombe M, Gill PMW, Johnson B, Chen W, Wong MW, Gonzalez C, Pople JA (2003) Gaussian 03, Revision B.04. Gaussian, Inc., Pittsburgh

35. Gómez-Jeria JS (2014) D-Cent-QSAR, a program to generate local atomic reactivity indices from Gaussian $03 \log$ files. V.1.0, Santiago de Chile

36. Gómez-Jeria JS (2009) An empirical way to correct some drawbacks of Mulliken population analysis (Erratum in: J. Chil. Chem. Soc., 55, 4, IX, 2010). J. Chil. Chem. Soc. 54: 482-485

37. StatSoft, Inc. (2011) STATISTICA (data analysis software system), version 10. www.statsoft.com

Publisher's note Springer Nature remains neutral with regard to jurisdictional claims in published maps and institutional affiliations. 